\title{
Contour Extraction based on Circular Hough Transform for Forest Canopy Digital Hemispherical Photography
}

\author{
Liangkuan Zhu, Liang Liu, Weipeng Jing ${ }^{*}$, Jianping Huang \\ College of Mechanical and Electrical Engineering, Northeast Forestry University, Harbin, 150040, China
}

\begin{abstract}
Analysis of forest canopy hemisphere images is one of the most important methods for measuring forest canopy structure parameters. This paper focuses on improvements to contour extraction method for the circular imaging region in forest canopy. We proposed an effective contour extraction algorithm based on circular hough transform for the circular imaging region of digital canopy hemispherical photography (DHP). In order to eliminate the threshold segmentation error, the morphological opening and closing was used to remove these desultory points and smooth the outline of the imaging region. After the boundary points of the imaging area were extracted through erosion, the circular hough transform was applied to fit the boundary point for finding the position of the center and gaining the radius. The experimental results proved its feasibility in terms of effective implementation and robustness.
\end{abstract}

Keywords: forest canopy; digital hemispherical photography (DHP); contour extraction; circular hough transform

(Submitted on September 29, 2017; Revised on November 2, 2017; Accepted on December 21, 2017)

(C) 2018 Totem Publisher, Inc. All rights reserved.

\section{Introduction}

Forest canopy characteristics are important in various applications, such as the conservation and restoration of understory species [2], invasive species control, post-tsunami assessment and analysis [20], acceleration of hurricane recovery, monitoring of water and carbon cycles [16,24], and radioecology [14]. Forest canopy characteristics include many physical and measurable variables such as canopy closure [18], canopy coverage, diffuse non-interceptance (DIFN) [23], gap fraction [21], leaf area index [19], light metering, etc. (Table 1). Among these various methods for measuring forest canopy variables, digital hemispherical photography (DHP) is widely used, because of its ease of use, quick acquisition, and low cost of acquisition [5].

Table 1. Common forest canopy variables and their definitions

\begin{tabular}{|l|l|}
\hline Variable & Physical measurement \\
\hline Canopy closure & Proportion of sky blocked by canopy at all angles from a single point \\
\hline Canopy coverage & Proportion of sky blocked by canopy at vertical angle from multiple points \\
\hline Diffuse non-interceptance & Amount of light, both direct and indirect, received from the sky \\
\hline Gap fraction & Proportion of open space in a canopy \\
\hline Leaf area index & Amount of foliage that a vertical line through the canopy will intersect \\
\hline Light metering & Amount of photonic energy from the sun \\
\hline
\end{tabular}

DHP is to estimate forest canopy characteristics by analysing photographs using a hemispherical fisheye lens with a $180^{\circ}$ circular view, which produces a circular projection of the forest canopy (Figure 1) [13]. Because of the large angle of fisheye lens, DHP provides more information than the non-hemispherical photography. First, the forest canopy is shot by hardware which commonly consists of a camera with a fisheye lens and optional accessories (tripod, leveling mount, and remote controller). Hemisphere images are stored commonly in compressed JPEG image format in the camera. Then, these images are digitally processed to differentiate sky from canopy. Gap fraction and LAI can be obtained by analyzing the 
relationship between the sky and the number of pixels in the canopy [25]. Other forest canopy parameters cn be obtained in a similar way. DHP has the greatest potential for canopy structure parameter estimation [9,25] and has the advantages of low sensitivity to illumination conditions, finer directional spatial sampling of canopy gaps, and ability to derive solar radiation regimes [4].

However, because the imaging region is circular, there are massive non-imaging pixels in the rectangular photograph from DHP. Figure 2 is a canopy hemisphere image collected in the forest, where the black pixels outside the circular imaging area are non-imaging pixels. Estimating the canopy parameters requires analysis of the sky portion and the canopy portion in the imaging region, so it is necessary to remove the non-imaging pixels to extract the imaging region. There are many advantages to extracting imaging areas:

- Because canopy analysis often requires precise parameter values, image processing algorithms that extract parameters usually are complex. It can reduce the amount of computation and the run time that pre-separating a large number of non-imaging pixels.

- After splitting part of imaging area of the sky and the canopy, it is easy to get another part.

Since the imaging area is circular, it is only necessary to extract the circular contour of the imaging area, i.e. the circular imaging region's center and radius. In Figure 2, the center of circular imaging region and the center of photograph are obviously not the same point, and the radius of circular imaging region is independent of the size of photograph. On the other hand, we know that the imaging range of a photograph is decided not only by the optical center of the camera but also by the focal length; different cameras with different fisheye lenses have different imaging ranges. Most importantly, the radius and the center cannot be found in the hardware specifications [22]. Therefore, imaging region's center and radius cannot be easily obtained by image size and hardware parameters, and should be calculated by image processing algorithms. This paper focuses on improvements to contour extraction method for the circular imaging region in forest canopy.

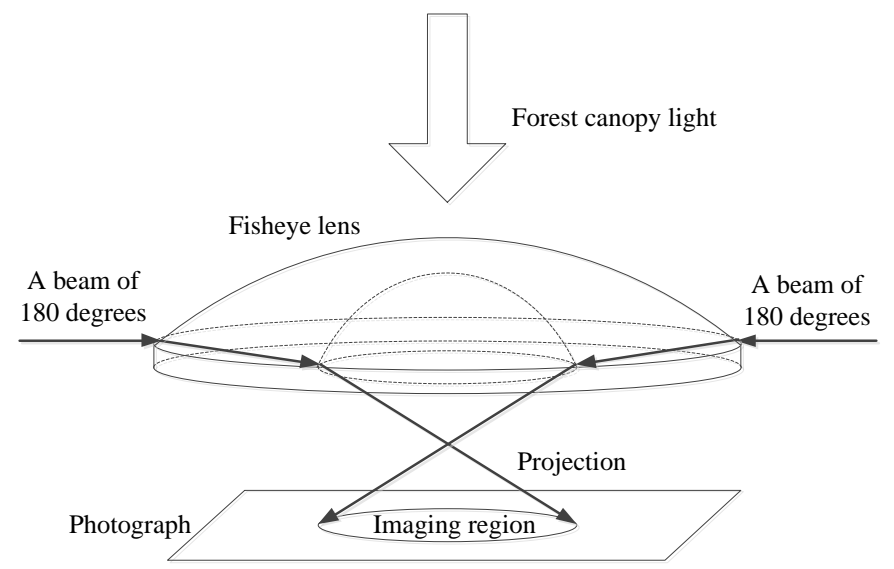

Figure 1. Imaging diagram of DHP

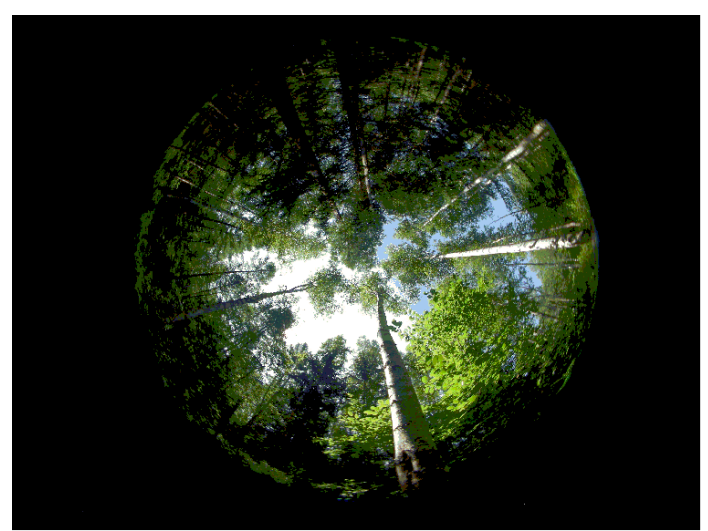

Figure 2. Forest canopy digital hemispherical image 
As far as we know, the literature about the contour extraction method for the circular imaging region in forest canopy digital hemispherical photography is less. Song et al. proposed a segmentation algorithm by the least squares method to fit the circular boundary and compute the center and radius of the circular imaging region [22]. They applied the algorithm to ideal images acquired under certain conditions in order to have a clear boundary and obtained good results. However, the environmental conditions of forest are complex and varied. Changes in light and other factors affect the extraction of the boundary point, leading to a bad result that some of the extracted edge information was wrong. Moreover, this often happens. The robustness of least squares method is not strong. These erroneous edge information often affect the accuracy of the algorithm, resulting in a larger error.

The Hough transform (HT) is a powerful tool in shape (curve like circle, line, and ellipse) analysis [6,15]. The HT was introduced by Paul Hough [7] in a patent filed in 1962. The basic principle of the HT is to project the image data within a parameter space representing possible positions of a shape in the image space, then to search accumulation points in the parameter space, corresponding to the most probable positions of the shape in the image [3]. The circular hough transform (CHT) is an application of the HT for circle detection [8]. Besides, CHT has strong robustness against noise, occlusions and discontinuities in images [12,17]. It can not only identify the circle whose part of the edge is missing, but can also resist the interference of the wrong boundary. Thus, it is ideal to reduce the impact of error boundary information and suitable for extracting circular contours in complex and changing environmental conditions.

The goal of this study is to propose an effective contour extraction algorithm based on circular hough transform for the circular imaging region, which could provide technical support for the follow process about estimating forest canopy characteristics in DHP.

\section{Materials and methods}

\subsection{Acquisition of hemispherical photographs}

The study site is located within the Liangshui National Nature Reserve, in Northeastern China. We used a Nikon Coolpix 4500 digital camera equipped with a calibrated FC-E8 fish-eye lens with a field of view of $180^{\circ}$ for all hemispherical photographs [11]. All of the hemispherical photographs of sample points were taken $1.3 \mathrm{~m}$ above the ground using a tripod, and the camera was leveled to face exactly the vertical using a bubble-level. We chose the following settings for the camera:

- $\quad$ Aperture priority mode with aperture set at F5.3 (i.e., automatic exposure).

- $3264 \times 2448$ Pixels image.

- $\quad$ Fine JPEG format.

A total of 29 hemispheric photographs were obtained at the sample points that were located random 29 points in the study site.

\subsection{Circle hough transforms}

As is the case for all Hough transforms, the CHT seeks to map an image into a quantized parameter space that describes the target objects. An arbitrary circle in two dimensions can be described by

$$
\left(x-x_{0}\right)^{2}+\left(y-y_{0}\right)^{2}-r^{2}=0
$$

where $x_{0}$ and $y_{0}$ are the $x$ and $y$ coordinates of the centre of the circle and $r$ is the circle's radius. Each different threetuple $\left(x_{0}, y_{0}, r\right)$ uniquely parametrizes different circles that may be present in an image. Each point in the Hough space therefore corresponds to a complete circle with a particular location and size.

In its common form, the Hough transform operates on each image pixel independently. For each pixel the algorithm finds the set of parameter tuples that would result in an object passing through the considered pixel. That is, consideration of an image pixel $f\left(x_{i}, y_{i}\right)$ finds the set $\Omega$ of all $\left(x_{0}, y_{0}, r\right)$ tuples that satisfy Equation 2 : 


$$
\Omega=\left\{\left(x_{0}, y_{0}, r\right):\left(x_{i}-x_{0}\right)^{2}+\left(y_{i}-y_{0}\right)^{2}-r^{2}=0\right\}
$$

Figure 3 illustrates the voting process. Points $\mathrm{P}_{1}, \mathrm{P}_{2}$ and $\mathrm{P}_{3}$ are taken randomly and belong to the same circle with $\mathrm{r}_{0}$ the radius and $\left(\mathrm{a}_{0}, \mathrm{~b}_{0}\right)$ the coordinates of its center. In the Hough space, each point generates a circular cone, i.e. the set $\Omega$ of parameter tuples that each point corresponds to. The three cones in the Hough space intersect at $\left(\mathrm{a}_{0}, \mathrm{~b}_{0}, \mathrm{r}_{0}\right)$ corresponding to the parameters of the circle formed by the points $\mathrm{P}_{1}, \mathrm{P}_{2}$ and $\mathrm{P}_{3}$.

For the application in this article, the algorithm constructs an accumulative matrix, based on votes in the Hough space by $\mathrm{CHT}$, and finds local maximums, then the radius and center of any circular features in the input image can be recovered.

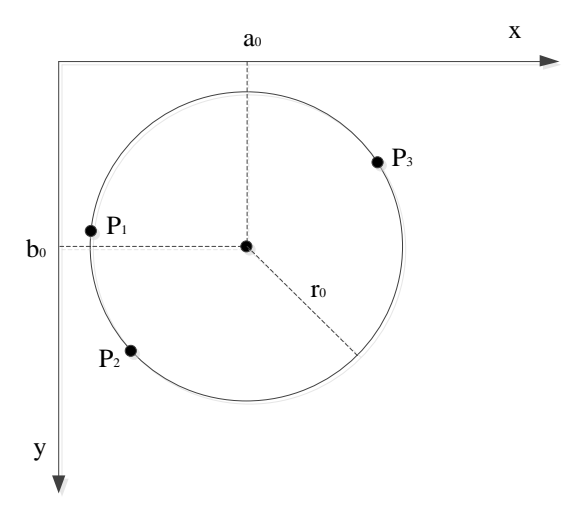

(a)

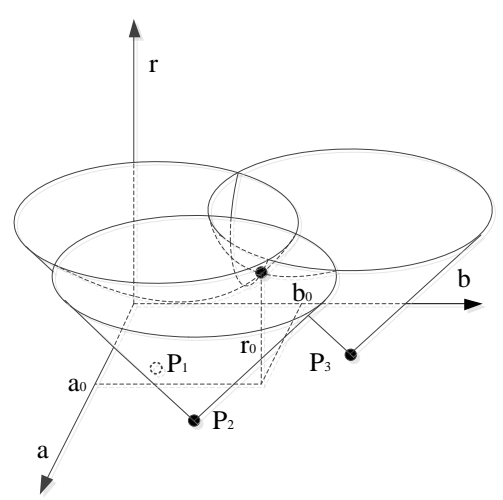

(b)

Figure 3. The voting process in CHT: (a) the input image; (b) the Hough space

\subsection{CHT-based contour extraction in DHP}

Image processing, calculation and analysis of contour extraction were implemented in Matlab 8.3 (R2014a) (The MathWorks, Inc.). The entire process of contour extraction algorithm is divided into two parts: boundary point extraction and circular boundary fitting. The aim of boundary point extraction is to get the edge information of the circular imaging region in the hemispherical photographs and provide support for the later process, i.e. circular boundary fitting. In circular boundary fitting, edge information is transformed into the Hough space by CHT, called the voting process. Then, the maximum is determined, and the center and radius that the peak represents are the circular imaging region's center and radius.

\subsection{Boundary point extraction}

In the hemispherical photograph from forest canopy, most are low green vegetation or branches of distant trees near the edge of circular imaging region. That is, the pixel values near the edge of the circular region are higher on the green component than the other two components. Thus, the proposed method uses the green component grayscale image of the hemisphere image to extract the edge information. We use minimum error thresholding to divide the green component grayscale image of the hemisphere image for obtaining the imaging region and the non-imaging area. Figure 4(a) is a binary image obtained by the thresholding processing the green component gray image of Figure 2, the white region being the imaging area, and the black region being the non-imaging area.

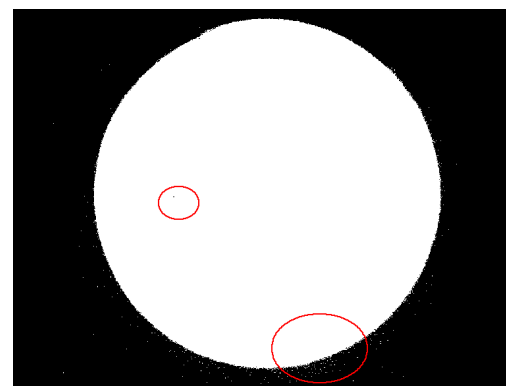

(a)

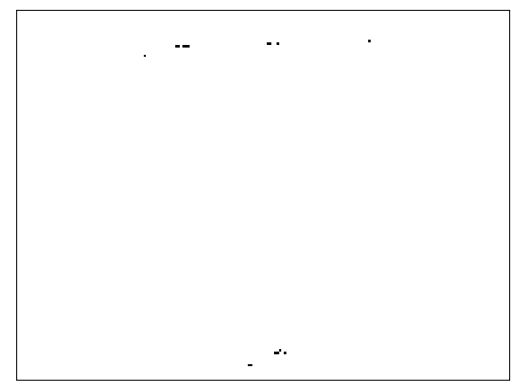

(b)

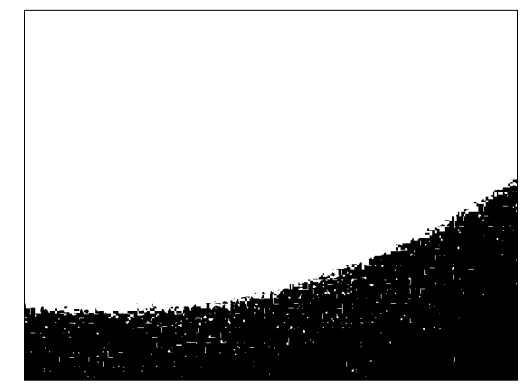

(c)

Figure 4 . The binary image obtained by minimum error thresholding and enlarge image 
The desultory points (some small white spots in black region and black spots in white region) usually appear in the result of canopy hemispherical image using minimum error thresholding, as shown in Figure 4(b)(c) as the enlarged figures of Figure 4(a) at the red circles. These points affect the extraction of the imaging area boundaries. The morphological opening and closing is used to remove these desultory points. The opening could remove the particular image detail that is smaller than the structural elements (i.e. white spots), and the closing could fill the particular holes that are smaller than the structural elements (i.e. remove black spots). They smooth the outline of the object, and at the same time ensure that it does not produce global geometric distortion $[1,10]$.

Let the imaging region be represented by a set $A$ whose elements are a tuple of the form $(x, y)$, where $x$ and $y$ are coordinates of a white pixel in the binary image. Let the flat circular structuring elements be represented by a set $B$, whose radius is 5 and center is at the origin. The opening of set $A$ by structuring element $B$, denoted by $A \circ B$, get the set $A^{\prime}$. It is defined as follows:

$$
A^{\prime}=A \circ B=(A \odot B) \oplus B
$$

where $\ominus$ represents the erosion. The erosion of set $P$ by $Q$, denoted by $P \ominus Q$, is defined as follows:

$$
\begin{gathered}
P \in Q=\left\{z \mid(Q)_{z} \cap P^{c}=\varnothing\right\} \\
(Q)_{z}=\{c \mid c=a+z, a \in Q\} \\
P^{c}=\{w \mid w \notin P\}
\end{gathered}
$$

where $\varnothing$ represents an empty set, $Q$ is a structuring element. In Equation (3), $\oplus$ represents the dilation. The dilation of set $P$ by $Q$, denoted by $P \oplus Q$, is defined as follows:

$$
\begin{gathered}
P \oplus Q=\left\{z \mid(\hat{\mathrm{Q}})_{z} \cap P \neq \varnothing\right\} \\
\hat{Q}=\{w \mid w=-b, b \in Q\}
\end{gathered}
$$

where $\varnothing$ represents an empty set, $Q$ is a structuring element. The closing of set $A^{\prime}$ by structuring element $B$, denoted by $A^{\prime} \bullet B$, get the set $A^{\prime \prime}$. The closing operation is opposite to the opening operation. The closing $A^{\prime}$ by $B$ is the dilation of $A^{\prime}$ by $B$, followed by a erosion of the result by $B$. It is defined as follows:

$$
A^{\prime \prime}=A^{\prime} \bullet B=\left(A^{\prime} \oplus B\right) \ominus B
$$

where $\oplus$ represents the dilation, and $\ominus$ represents the erosion. The result of the Figure 4 applying opening and closing is shown in Figure 5, and the set of all white pixels in the image is $A^{\prime \prime}$.

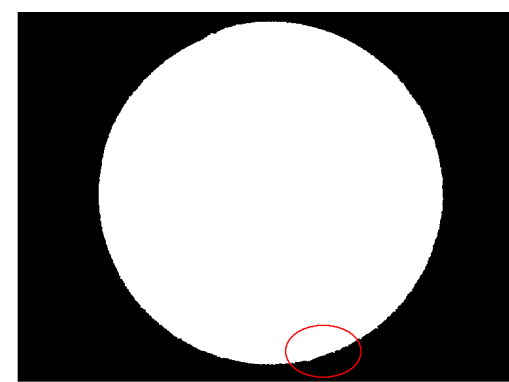

(a)

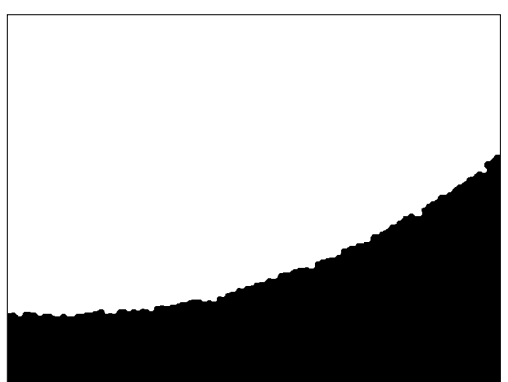

(b)

Figure 5. (a)The result image using opening and closing; (b) the enlarged figures of (a) at the red circle 
Finally, by extracting the boundary of the set $A^{\prime \prime}$ to get the contour information of the imaging region. The boundary of set $A^{\prime \prime}$, denoted by $\beta\left(A^{\prime \prime}\right)$, can be obtained by first eroding $A^{\prime \prime}$ by $C$ and then performing the set difference between $A^{\prime \prime}$ and its erosion. That is,

$$
\beta\left(A^{\prime \prime}\right)=A^{\prime \prime}-\left(A^{\prime \prime} \odot C\right)
$$

where $C$ is the square structuring element whose length of side is 3 . Figure 6 shows the result of boundary point extraction. For clearly showing, the border width in the image has been enlarged.

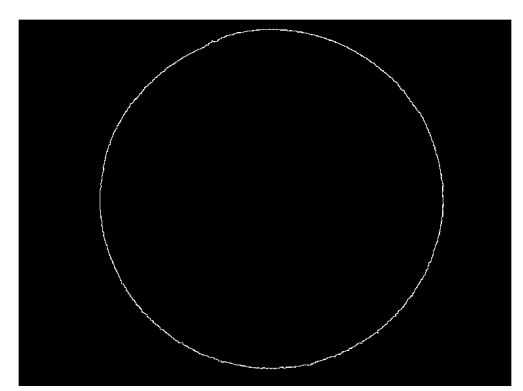

Figure 6. Boundary point extraction

\subsection{Circular boundary fitting}

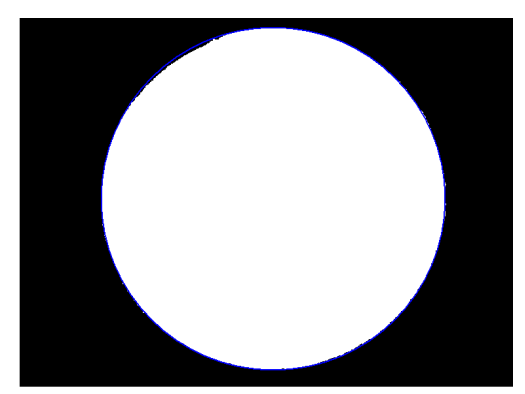

Figure 7. The fitting circle

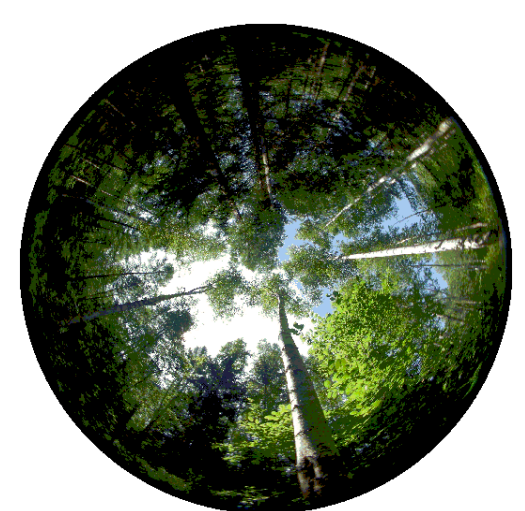

Figure 8. The circular imaging region

In circular boundary fitting, the algorithm completes the contour extraction of the circular imaging area based on the edge information obtained from the boundary point extraction. The important aims of this process are to find the position of the center and obtain the radius accurately. In order to increase the performance of CHT, we resize all images so that the length and width become $1 / 4$ of the original and search for the circle with an experimented selected radius range of 250-306 pixels. The boundary points gained from the previous are transformed by CHT. Among circular responses generated by CHT, only the strongest circle must be chosen. The strongest circle is the one that correspond to the contour of the circular area. To test the effectiveness of the above process, they were applied to Figure 6 and obtained that the radius of the fitting circle was 284 pixels and center coordinate was $(419,300)$. Since the resizing was done before CHT, the true radius and the center coordinate of the circle are four times the resulting value, and the true radius is 1136 pixels and center coordinate is (1676, 
1200). In order to show the fitting effect more clearly, the circle is plotted with blue lines according to the resulting radius and center in Figure 5 (Figure 7). The results show that the above process can fit the circular boundary well, and they are effective. Finally, the circular imaging region obtained by the proposed contour extraction algorithm is shown in Figure 8.

\section{Results and discussion}

In order to reflect the good performance of the proposed contour extraction algorithm, it was compared with the contour extraction algorithm based on least squares method (LS extraction algorithm). In the experiment, 29 hemispheric images were analyzed by the method in this paper and LS extraction algorithm, and the results were then compared with the manual measurement.

The step of the manual method (MM) is as follows:

- Step 1. Threshold segmentation and morphological processing were applied to the target image to get the imaging area.

- Step 2. In Photoshop 14.0 (Adobe Systems Incorporated), the circle was drawn on the imaging area by the ellipse marquee tool. The size and position were then adjusted by the transforming selection so that the circular selection is best suited for the imaging area.

- Step 3. The vertex coordinates of the circumscribed square of the circular area were read, and the radius and center coordinates were calculated.

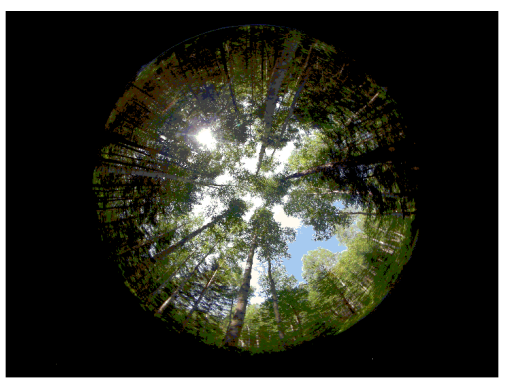

(a)

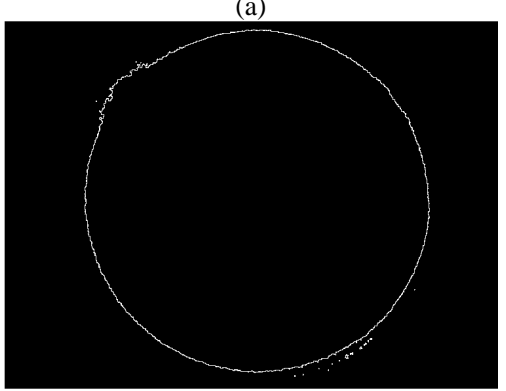

(d)

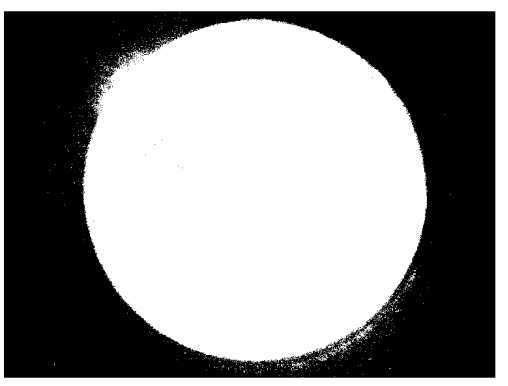

(b)

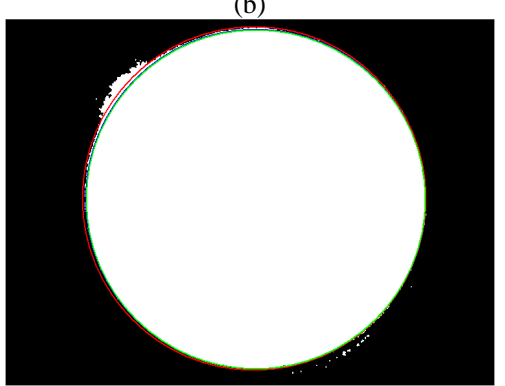

(e)

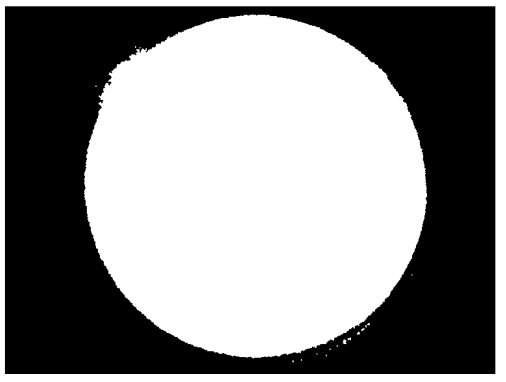

(c)

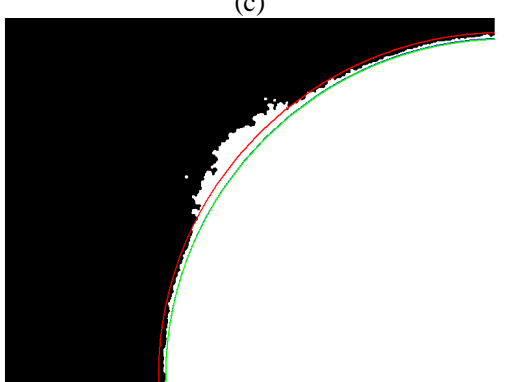

(f)

Figure 9. Forest canopy hemisphere image one and its processing results: (a) the original image; (b) the result of the threshold segmentation; (c) the result of the morphological operation; (d) the extracted boundary point; (e) the fitting circles (red for LS, blue for proposed method, green for MM); (f) the enlarged figure

Among the processed hemisphere images, two representative samples were analyzed in detail. In Figure 9, it can be seen that most of the circle of LS extraction algorithm was consistent with the circle of manual method, but the circle of LS algorithm was slightly larger in the upper right corner, while the circles obtained by the proposed method and the manual method almost coincided. In short, in this case the proposed method is superior to the LS extraction algorithm.

Figure 10 reflects the robustness of the proposed method under complex and changing environmental conditions. In Figure 10, it can be seen that the circle fitted by the LS extraction algorithms seriously deviated from the correct circle, while the circle of the proposed method had little difference from the correct circle. In summary, the method of this paper has stronger robustness and provides higher recognition accuracy.

Table 2 shows the average error of the collected 29 hemispherical images through LS extraction algorithm and the proposed method. The center error is the distance between the obtained and manual method's center coordinates. The radius error is the absolute value of the difference between the obtained and manual method's radius. The total error is the sum of 
the center error and the radius error. It can be calculated that the average center error of LS extraction algorithm is 5.8 times that of the proposed method, and the average radius error is 6.1 times that of the proposed method. The average total error is 5.9 times that of the proposed method.

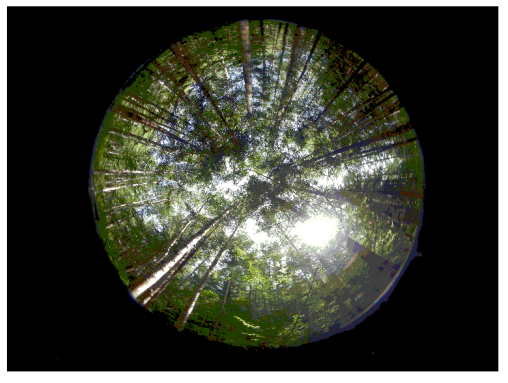

(a)

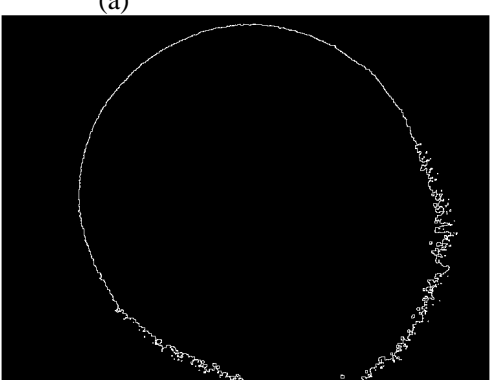

(d)

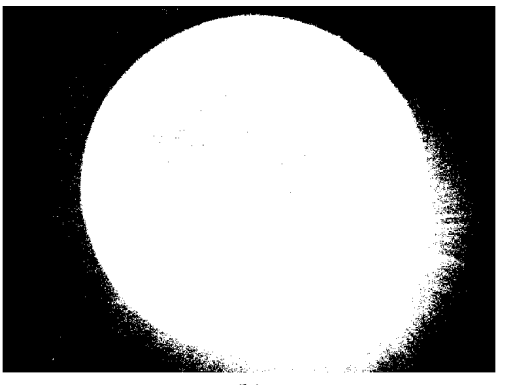

(b)
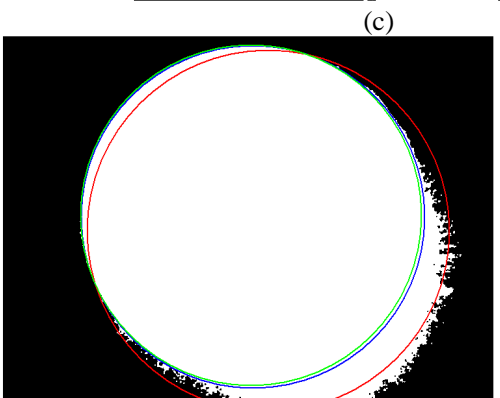

(e)

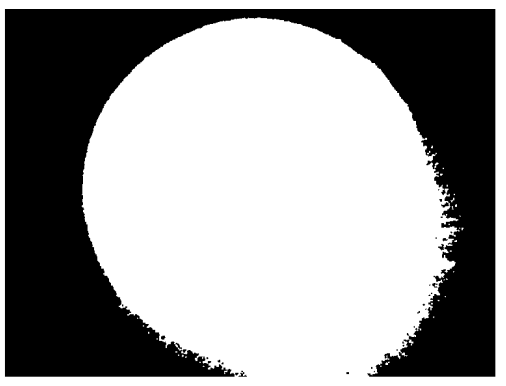

Figure 10. Forest canopy hemisphere image two and its processing results: (a) the original image; (b) the result of the threshold segmentation; (c) the result of the morphological operation; (d) the extracted boundary point; (e) the fitting circles (red for LS, blue for proposed method, green for MM)

Table 2. The average error of the LS extraction algorithm and proposed method (unit: pixel)

\begin{tabular}{|c|c|c|}
\hline Average error & LS & Proposed \\
\hline Center error & 33.82 & 5.84 \\
\hline Radius error & 17.31 & 2.86 \\
\hline Total error & 51.13 & 8.70 \\
\hline
\end{tabular}

\section{Conclusions}

In this paper, we proposed a method of contour extraction based on circular Hough transform. The algorithm used minimum error thresholding to divide the green component grayscale image of the hemisphere image and obtain the imaging region and the non-imaging area. This approach can reduce the effect of interference generated under complex and changing environmental conditions. Considering the running time and computational complexity, the method of this article could reduce the size of the original image and set the a priori information of the radius range. Hence, this study lays a foundation for automatically adjusting the parameters of high-performance image acquisition devices used in forest canopy hemispherical photography.

\section{Acknowledgements}

The authors are grateful for the support of the National Natural Science Foundation of China (31370710), Forestry Science and Technology Extension Project (2016[34]), the 948 Project (2014-4-46), the Fundamental Research Funds of Central Universities (DL12EB04-02), and the Postdoctoral Research Fund of Heilongjiang Province (LBH-Q13007).

\section{References}

1. X. Bai, "Morphological Infrared Image Enhancement Based on Multi-scale Sequential Toggle Operator Using Opening and Closing as Primitives," Infrared Physics \& Technology, vol. 68, pp. 143-151, January 2015

2. A. Brusa and D. E. Bunker, "Increasing the Precision of Canopy Closure Estimates from Hemispherical Photography: Blue Channel Analysis and Under-exposure," Agricultural And Forest Meteorology, vol. 195, pp. 102-107, September 152014

3. A. O. Djekoune, K. Messaoudi, and K. Amara, "Incremental Circle Hough Transform: An Improved Method for Circle Detection," Optik, vol. 133, pp. 17-31, March 2017

4. S. Garriques, N. V. Shabanov, K. Swanson, J. T. Morisette, F. Baret, and R. B. Myneni, "Intercomparison and Sensitivity Analysis of Leaf Area Index Retrievals from LAI-2000, AccuPAR, and Digital Hemispherical Photography over Croplands," Agricultural And Forest Meteorology, vol. 148, no. 8-9, pp. 1193-1209, July 42008 
5. J. Glatthorn and P. Beckschafer, "Standardizing the Protocol for Hemispherical Photographs: Accuracy Assessment of Binarization Algorithms," Plos One, vol. 9, no. 11, November 2014

6. I. Grishin, K. Thomson, F. Migliorini, and J. J. Sloan, "Application of the Hough Transform for the Automatic Determination of Soot Aggregate Morphology," Applied Optics, vol. 51, no. 5, pp. 610-620, February 102012

7. V. P. C. Hough, "Method and Means for Recognizing Complex Patterns," U.S., December 1962

8. J. Illingworth and J. Kittler, "A Survey of the Hough Transform," Computer vision, graphics, and image processing, vol. 44, no. 1 ,pp. 87-116, 1988

9. I. Jonckheere, S. Fleck, K. Nackaerts, B. Muys, P. Coppin, M. Weiss, et al., "Review of Methods for in Situ Leaf Area Index Determination - Part I. Theories, Sensors and Hemispherical Photography," Agricultural And Forest Meteorology, vol. 121, no. 1-2, pp. 19-35, January 2004

10. K. Kwak, U. Yoon, D.-K. Lee, G. H. Kim, S. W. Seo, D. L. Na, et al., "Fully-automated Approach to Hippocampus Segmentation Using a Graph-cuts Algorithm Combined with Atlas-based Segmentation and Morphological Opening," Magnetic Resonance Imaging, vol. 31, no. 7, pp. 1190-1196, September 2013

11. Z. Liu, J. M. Chen, G. Jin, and Y. Qi, "Estimating Seasonal Variations of Leaf Area Index Using Litterfall Collection and Optical Methods in four Mixed Evergreen-deciduous Forests," Agricultural And Forest Meteorology, vol. 209, pp. 36-48, September 152015

12. R.-C. Lo and H.-C. Hsu, "A Circular Band Extraction Method Based on Extended Hough Transform," International Journal Of Pattern Recognition And Artificial Intelligence, vol. 30, no. 8, September 2016

13. N. Loffredo, X. C. Sun, and Y. Onda, "DHPT 1.0: New Software for Automatic Analysis of Canopy Closure from Underexposed and Over-exposed Digital Hemispherical Photographs," Computers and Electronics in Agriculture, vol. 125, pp. 39-47, July 2016

14. N. Loffredo, Y. Onda, P. Hurtevent, and F. Coppin, "Equation to Predict the Cs-137 Leaching Dynamic from Evergreen Canopies after a Radio-cesium Deposit," Journal Of Environmental Radioactivity, vol. 147, pp. 100-107, September 2015

15. A. Manzanera, N. Thanh Phuong, and X. Xu, "Line and Circle Detection Using Dense one-to-one Hough Transforms on Greyscale Images," Eurasip Journal on Image And Video Processing, December 122016

16. F. J. Mao, X. J. Li, H. Q. Du, G. M. Zhou, N. Han, X. J. Xu, et al., "Comparison of Two Data Assimilation Methods for Improving MODIS LAI Time Series for Bamboo Forests," Remote Sensing, vol. 9, no. 5, p. 17, May 2017

17. M. Mirzaei and H. K. Rafsanjani, "An Automatic Algorithm for Determination of the Nanoparticles from TEM Images Using Circular Hough Transform," Micron, vol. 96, pp. 86-95, May 2017

18. J. R. Parent and J. C. Volin, "Assessing the Potential for Leaf-off LiDAR Data to Model Canopy Closure in Temperate Deciduous Forests," Isprs Journal Of Photogrammetry And Remote Sensing, vol. 95, pp. 134-145, September 2014

19. C. Poblete-Echeverria, S. Fuentes, S. Ortega-Farias, J. Gonzalez-Talice, and J. A. Yuri, "Digital Cover Photography for Estimating Leaf Area Index (LAI) in Apple Trees Using a Variable Light Extinction Coefficient," Sensors, vol. 15, no. 2, pp. 2860-2872, February 2015

20. P. R. C. Prasad, N. Nagabhatla, C. S. Reddy, S. Gupta, K. S. Rajan, S. H. Raza, et al., "Assessing Forest Canopy Closure in a Geospatial Medium to Address Management Concerns for Tropical Islands-Southeast Asia," Environmental Monitoring And Assessment, vol. 160, no. 1-4, pp. 541-553, January 2010

21. T. Sasaki, J. Imanishi, K. Ioki, Y. Song, and Y. Morimoto, "Estimation of Leaf Area Index and Gap Fraction in two Broadleaved Forests by Using Small-footprint Airborne LiDAR," Landscape And Ecological Engineering, vol. 12, no. 1, pp. 117-127, January 2016

22. J.-y. Song, W.-1. Song, J.-p. Huang, and L.-k. Zhu, "Segmentation and Focus-point Location based on Boundary Analysis in Forest Canopy Hemispherical Photography," Frontiers Of Information Technology \& Electronic Engineering, vol. 17, no. 8, pp. 741-749, August 2016

23. F. Tinya, B. Mihok, S. Marialigeti, Z. Mag, and P. Odor, "A Comparison of three Indirect Methods for Estimating Understory Light at Different Spatial Scales in Temperate Mixed Forests," Community Ecology, vol. 10, no. 1, pp. 81-90, January 2009

24. T. L. Zhang, R. Sun, C. H. Peng, G. Y. Zhou, C. L. Wang, Q. A. Zhu, et al., "Integrating a Model with Remote Sensing Observations by a Data Assimilation Approach to Improve the Model Simulation Accuracy of Carbon Flux and Evapotranspiration at two Flux Sites," Science China-Earth Sciences, vol. 59, no. 2, pp. 337-348, February 2016

25. K. N. Zhao and F. L. He, "Estimating Light Environment in Forests with a New Thresholding Method for Hemispherical Photography," Canadian Journal of Forest Research, vol. 46, no. 9, pp. 1103-1110, September 2016 